\title{
Challenges of New Approaches in Metastatic Merkel Cell Carcinoma
}

\author{
Ana Monteiro $^{a} \quad$ Emanuel Gouveia ${ }^{a} \quad$ Daniela Garcez $^{b}$ Sara Donato ${ }^{c}$ \\ Diogo Martins-Branco ${ }^{a}$ Joana Marques ${ }^{a}$ Hugo Nunes ${ }^{a}$ \\ Maria José Passos ${ }^{a} \quad$ Ana Isabel Clara ${ }^{a}$ António Moreira ${ }^{a}$ \\ a Medical Oncology Department, Instituto Português de Oncologia de Lisboa Francisco \\ Gentil, E.P.E, Lisbon, Portugal; beurology Department, Instituto Português de Oncologia

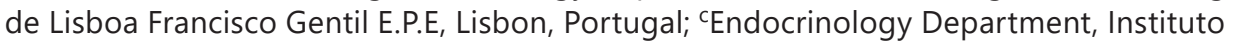 \\ Português de Oncologia de Lisboa Francisco Gentil, E.P.E, Lisbon, Portugal
}

\author{
Keywords \\ Immunotherapy · Merkel cell carcinoma $\cdot$ Immune-related adverse events · Diabetic \\ ketoacidosis $\cdot$ Pan-cerebellar syndrome
}

\begin{abstract}
Merkel cell carcinoma is a rare and aggressive cutaneous tumor, and the use of checkpoint inhibitors immunotherapy is a recent indication in its metastatic setting, both first and second line. However, the widespread use of immunotherapy is associated with an increase of acute and late immune-mediated adverse events. We present a case of an elderly fit patient with metastatic Merkel cell carcinoma treated with pembrolizumab who developed diabetic ketoacidosis, a severe immune-mediated adverse event. A multidisciplinary approach was crucial to overcome the life-threatening event. Even with early treatment stop, the patient had a significant and durable response to the treatment for 15 months. Meanwhile, a progressive pancerebellar syndrome emerged, possible due to a paraneoplastic syndrome with a negative onco-neuronal antibody panel, although an autoimmune etiology associated with immunotherapy could not be excluded. Unfortunately, the situation was irreversible and refractory to immunomodulatory treatment. Despite the unpredictable toxicity, it is important to note the efficacy profile, with a progression-free survival of 15 months, which is higher than the one reported in reference clinical trials in this setting.


Monteiro et al.: Approaching Metastatic Merkel Cell Carcinoma

\section{Introduction}

Merkel cell carcinoma (MCC) is a rare and aggressive cutaneous tumor with neuroendocrine differentiation. Its incidence tripled in the last 20 years due to aging and also due to diagnostic accuracy improvement (routine use of immunohistochemical marker CK-20). A large meta-analysis showed that nearly one-half of MCC develop lymph node metastasis and nearly one-third present with distant metastasis [1]. Mortality rate is higher than with melanoma, with important morbidity associated with locoregional disease [2].

For many years, chemotherapy has been the backbone treatment of recurrent or metastatic MCC. This therapeutic approach was associated with favorable initial response, albeit short lived and without significant overall survival benefit [2, 3].

The use of immune checkpoint inhibitors (ICI) immunotherapy is a recent indication for the treatment of metastatic MCC, both first and second line. In the USA, the Food and Drugs Administration (FDA) approved avelumab and, more recently, pembrolizumab in this setting. In Europe, avelumab (but not pembrolizumab) is approved as monotherapy for the treatment of adult patients with metastatic MCC. The published data in different trials with ICI in this setting showed objective response rates from 56 to $62 \%$.

When the patient presented with metastatic MCC at our Dermatology and Oncology Department, we were aware of the first results of a clinical trial with pembrolizumab reported in the New England Journal of Medicine and decided to propose this treatment as an off-label indication. This phase 2, single-arm, and multicentric trial included 50 patients who were treated with pembrolizumab, showing an objective response rate of 56\%. Most responses (96\%) were sustained (more than 6 months), and 54\% lasted more than 12 months [4]. However, a longer follow-up is needed to evaluate the duration of response and progressionfree survival.

Although there are no comparative trials to demonstrate the superiority of immunotherapy over chemotherapy, response rates were similar to the ones previously reported with chemotherapy but long lasting.

With the increased use of immunotherapy, there is the emergence of a new spectrum of toxicities, including immune-mediated adverse events (AEs). Based on the results of preliminary trials, the toxicity profiles of patients with MCC were similar for avelumab, pembrolizumab, and nivolumab, with any AE occurring in $68-77 \%$ of patients and AE grade 3 or 4 in $5-21 \%$ of patients [4].

We present a clinical case of a patient with metastatic MCC treated with pembrolizumab who developed a diabetic ketoacidosis and subsequently a fatal cerebellar degeneration that emerged after treatment withdrawal.

\section{Case Report}

An 82-year-old Caucasian man (ECOG PS 0) with a personal history of hypertension and tobacco and alcohol consumption presented by the end of 2016 with progressive growth of axillary and cervical lymph nodes in the previous year. He underwent a biopsy that revealed MCC lymph node metastasis (immunohistochemistry profile: CK20+, CK7-, TTF1-, chromogranin+, synaptophysin+). The staging PET 68-Ga DOTANOC performed on January 20, 2017, reported supradiaphragmatic lymph node metastases not deemed eligible for surgery. After considering age and comorbidities, the patient was proposed for a treatment with pembrolizumab (2 mg/kg q3w) and started this treatment on May 2017, with objective clinical response after 4 cycles. At this point, the patient presented with acute anorexia and mental confusion, and he was referred to our Emergency Department. On admission, he was obnubilated and 
Fig. 1. Thoracic computed tomography before treatment start.

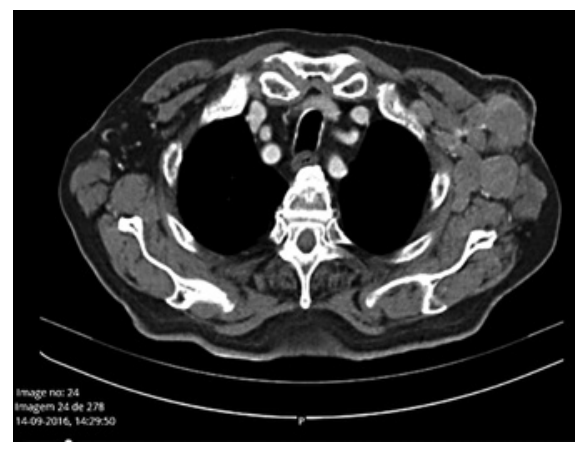

dehydrated. Blood analysis revealed hyperglycemia $(1,350 \mathrm{mg} / \mathrm{dL})$, acute kidney injury grade 3 associated with hyponatremia, hypercalcemia, hyperphosphatemia, and ketonuria $(20 \mathrm{mg} /$ $\mathrm{dL}$, normal range $0.3-3 \mathrm{mg} / \mathrm{dL}$ ). The clinical picture rapidly evolved with respiratory arrest with bradycardia and hypotension; he underwent orotracheal intubation and mechanic ventilation, and aminergic support was started. Initial blood gas analysis revealed mixed metabolic acidemia. He was then admitted to the Intensive Care Unit for advanced life support, and progressive clinical stability was observed. Complementary exams showed increased amylase and lipase but low $C$ peptide $(0.4 \mathrm{ng} / \mathrm{mL}$, normal range $0.9-7.1 \mathrm{ng} / \mathrm{mL})$. No anti-GAD, anti-TPO, and anti-Tg antibodies were found. The studies of the pituitary and thyroid function were normal. Diagnosis of diabetic ketoacidosis was established based on presentation of inaugural insulinopenic type 1 diabetes and possible pancreatitis due to pembrolizumab. Intensive insulin therapy and subsequent support therapy were started. At the sixth day after the ictus and after clinical stabilization, the patient was transferred to the Oncology Ward, and the clinical condition rapidly improved. Two weeks later, he was discharged under corticosteroid therapy at weaning and insulin therapy. Immunotherapy was discontinued and the patient kept under close surveillance at the Medical Oncology and Endocrinology Departments. Six months later, he was under basal bolus insulin therapy (30 U/day).

Between January and March 2018, he presented with an insidious and progressive worsening of dysarthria and ataxia, which prompted admission to our Oncology Ward for investigation. A chest-abdomen-pelvis computed tomography was performed for reassessment of the disease, and a substantial partial response was confirmed (only axillary lymph node metastasis persisted) (Fig. 1). The brain and neural-axis magnetic resonance imaging did not reveal any relevant findings, and the electromyography showed a mild axonal sensorimotor polyneuropathy. The laboratory work was positive only for ANA $(\geq 1 / 640$, fine granular pattern), and all the remaining analyses, including other markers for autoimmune diseases, vitamin assays, and viral and other serologies, were negative.

Lumbar puncture analysis found slight proteinorachia $(54 \mathrm{mg} / \mathrm{dL})$, absent pleocytosis, and intrathecal antibody synthesis, but with mirror banding profile suggesting systemic inflammation (pattern 4); the cytology was negative for malignant cells; the immunofluorescence assay of serum and cerebrospinal fluid showed a fine granular IgG staining of both granular and molecular layers of rat cerebellum. The subsequent analyses with immunoblot (anti-Hu, Ri, Yo, CV2, amphiphysin, PNMA2/Ma2/Ta, recoverin, SOX1, titin, Zic4, GAD65, Tr/ DNER, MAG) and with recombinant cells (anti-CASPR2, LGI1, receptors NMDA, AMPA1, AMPA2, GABAB) were negative. Samples were sent to the Clinical Immunology Laboratory (Lübeck, Germany) that completed the study with anti-AQ4, MOG, IgLON5, and DPPX; in the scope of research, the following antigens were also evaluated but have not yet been fully validated: CARPVIII, rGlicina, mGluR1, mGluR5, rGABA-A, GLURD2, flotillin, 26 activator protein of RhoGTPase, ITPR1, Hommer 3, and neurochondrin, which were also negative. 
Fig. 2. Upper digestive endoscopy showed gastric metastasis, confirmed by pathologic report, 15 months after ICI treatment start.

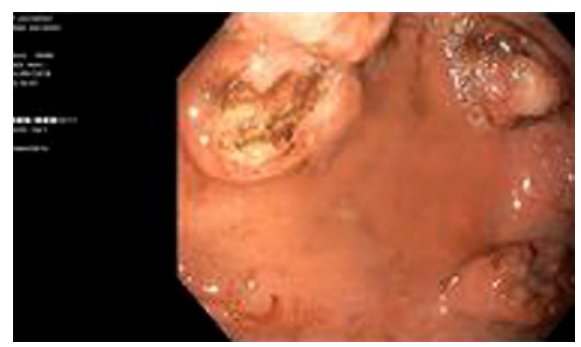

Despite lack of identification of any circulating antibody in serum and cerebrospinal fluid, we assumed the most likely hypothesis of immune-mediated cerebellar degeneration and started treatment with methylprednisolone pulse therapy ( $1 \mathrm{~g} /$ day for 5 days).

Three weeks later, the neurologic deficits worsened, mainly appendicular and axial ataxia, cerebellar dysarthria, and additionally dysphagia. The patient was readmitted to a new cycle of methylprednisolone with simultaneous administration of intravenous immunoglobulin $(0.4 \mathrm{mg} / \mathrm{kg}$ id) for 10 days. A new cycle of 5 days was repeated a few weeks later, but unfortunately, there was no evidence of clinical response.

The situation was discussed in the multidisciplinary tumor board, and the decision was to not proceed for plasmapheresis given the patient's general condition. Furthermore, it was likely that the circulating antibodies were not related to a pathogenic humoral response and were rather an epiphenomenon of the cytotoxic cellular response, more characteristic of this syndrome.

Due to worsening dysphagia, a nasogastric tube was placed under endoscopy guidance. During this procedure, agastric mucosa infiltration was detected, and biopsies were performed (Fig. 2). Histology was compatible with carcinoma CK20+ (in dot), NSE+, CD56+, chromogranin A+, synaptophysin+, and CD117+. Thus, progression of MCC was confirmed 15 months after starting immunotherapy. The progressive neurologic disability in the context of disease progression halted additional treatment other than palliative care; he passed away 1 month later.

\section{Discussion}

This case illustrates the particularity of an elderly patient with metastatic MCC with a significant response to immunotherapy after only 4 cycles of pembrolizumab; progressionfree survival was 15 months and overall survival was 16 months. However, this was associated with severe endocrine and neurologic events.

In a phase 2 trial with patients with metastatic MCC treated with pembrolizumab, grade 3 or 4 AEs were reported in $15 \%$ of the patients [4]. In this trial, 2 patients stopped the treatment and started corticosteroid, but both had sustained disease responses.

New onset of diabetes induced by ICI is rare $(0.1$ to $<1 \%$ in clinical trials); it can present with ketoacidosis, a rare but life-threatening event [5], and seems to be more frequent with anti-PD-1 and anti-PD-L1 inhibitors than with anti-CTLA4. A possible mechanism is due to the destruction of the $\beta$ islet cells of the pancreas by host $T$ cells which are no longer inhibited due to the effects of the PD-L1 therapy.

A review of type 1 autoimmune diabetes mellitus associated with pembrolizumab showed a median age of 58 years, no gender differences, and did not find any association with the number of treatment cycles [5-7].

In this case, as in others, we were unable to identify any potential risk factors, such as personal and family history, autoimmune disease, viral infections, liver metastasis, or toxics. 
Although rare, we also considered the hypothesis of pancreatitis given the clinical symptoms and amylase and lipase elevation. However, this could be just a consequence of diabetic ketoacidosis. In a systematic review including non-randomized clinical trials (early phase), an absolute risk of pancreatitis of 15\% was found. Nevertheless, among 3, 205 patients treated with anti-PD-1 in randomized controlled trials, there were no reported cases of pancreatitis [8]. In a lung cancer randomized controlled trial, the risk was reported to be $<1 \%$ (no grade 3-5) [9].

Neurologic complications of anti-PD-1 occur in 3\% of patients and mainly affect the peripheral nervous system, including myasthenia gravis, neuropathy, and myopathy. Central nervous system complications are rare and include aseptic meningitis, internuclear ophthalmoplegia, autoimmune encephalitis, transverse myelitis, and others. Most of these complications occur during immunotherapy treatment. However, some rare reports of immunemediated syndromes emerged a few months after immunotherapy stop [10,11]. Thus, it becomes difficult to distinguish between a pure paraneoplastic syndrome and acute or late neurologic toxicity of immunotherapy, and, in the literature, there is sometimes an arbitrary use of these terms. Nevertheless, we highlight the importance of restaging patients due to possible associated disease relapse. The presence of a negative antineuronal antibody panel does not exclude the presence of other antibodies not yet identified. The weak response to immunomodulator treatment is common between patients with neurologic paraneoplastic syndromes, including those with pan-cerebellar syndromes (cerebellar degenerescence), with a frequent dismal prognosis. Despite this, based on the existence of rare cases that respond to immunomodulatory treatment, some authors argue that this approach should be attempted. Unfortunately, in this case, the severity was impressive, and an irreversible clinical scenario developed, leading to the death of the patient within a few months.

\section{Conclusion}

With the increasing use of immunotherapy, it is important to be aware of potential AEs. We must keep in mind that there is a broad spectrum of immune-mediated events and that they can present with several grades. The AEs reported in this paper, although rare, highlight the need for a close monitoring, including, for example, serum glycemic levels and regular neurologic examination. In this case, despite the severity of the first endocrine AE, a multidisciplinary approach and admission to the Intensive Care Unit allowed complete recovery of the patient.

Regarding the neurologic event, the insidious evolution prevented a prompt diagnosis. Furthermore, the combined immunoglobulin and corticosteroid therapy did not halt the progression of deficits. Even if it could have been a late immuno-mediated AE of pembrolizumab, it is also not possible to exclude a paraneoplastic syndrome related to disease recurrence, potentially worsened or not by ICI. We emphasize that the negativity of the onconeuronal panels does not exclude the presence of unidentified antibodies. Despite the toxicity, it is important to note the efficacy profile, with a progression-free survival of 15 months, higher than the one reported in reference clinical trials in this setting.

\section{Acknowledgement}

We acknowledge the Medical Oncology Department, Neurology Department, and Endocrinology Department of Instituto Português de Oncologia de Lisboa Francisco Gentil, E.P.E. 
Monteiro et al.: Approaching Metastatic Merkel Cell Carcinoma

\section{Statement of Ethics}

The case presentation was conducted ethically in accordance with the World Medical Association Declaration of Helsinki. The son of the patient gave his consent to the publication of the case report relative to the clinical situation of the patient.

\section{Disclosure Statement}

The authors have no conflicts of interest to declare.

\section{Funding Sources}

There were no funding sources.

\section{Author Contributions}

Ana Monteiro: Substantial contributions to the conception or design of the work; drafting the work or revising it critically for important intellectual content; agreement to be accountable for all aspects of the work in ensuring that questions related to the accuracy or integrity of any part of the work are appropriately investigated and resolved. Emanuel Gouveia: Substantial contributions to the conception or design of the work; drafting the work or revising it critically for important intellectual content; final approval of the version to be published. Daniela Garcez: Substantial contributions to the conception or design of the work; drafting the work or revising it critically for important intellectual content. Sara Donato: Drafting the work or revising it critically for important intellectual content. Diogo MartinsBranco: Drafting the work or revising it critically for important intellectual content. Hugo Nunes: Drafting the work or revising it critically for important intellectual content. Maria José Passos: Drafting the work or revising it critically for important intellectual content; final approval of the version to be published. Joana Marques: Drafting the work or revising it critically for important intellectual content. Ana Isabel Clara: Drafting the work or revising it critically for important intellectual content. António Moreira: Drafting the work or revising it critically for important intellectual content.

\section{References}

1 Bichakjian CK, Olencki T, Aasi SZ, Alam M, Andersen JS, Blitzblau R, et al. Merkel Cell Carcinoma, Version 1.2018, NCCN Clinical Practice Guidelines in Oncology. J Natl Compr Cancer Netw. 2018 Jun;16(6) [Internet]. [cited 2019 Nov 16]. Available from: https://jnccn.org/view/journals/jnccn/16/6/article-p742.xml

2 Teixeira V, Vieira R, Julião MJ, Figueiredo A. Carcinoma de células de Merkel - normas de orientação clínica. J Port Soc Dermatol Venereol. 2013;71(1):23-32.

3 Lebbe C, Becker JC, Grob JJ, Malvehy J, del Marmol V, Pehamberger H, et al. Diagnosis and treatment of Merkel Cell Carcinoma. European consensus-based interdisciplinary guideline. Eur J Cancer. 2015 Nov;51(16):2396403.

4 Nghiem PT, Bhatia S, Lipson EJ, Kudchadkar RR, Miller NJ, Annamalai L, et al. PD-1 blockade with pembrolizumab in advanced Merkel-cell carcinoma. N Engl J Med. 2016 Jun 30;374(26):2542-52.

5 Maamari J, Yeung SJ, Chaftari PS. Diabetic ketoacidosis induced by a single dose of pembrolizumab. Am J Emerg Med. 2019 Feb;37(2):376-e2.

6 Cheema A, Makadia B, Karwadia T, Bajwa R, Hossain M. Autoimmune diabetes associated with pembrolizumab: a review of published case reports. World J Oncol. 2018 Mar 7;9(1):1-4. 
7 Li S, Zhang Y, Sun Z, Hu J, Fang C. Anti-PD-1 pembrolizumab induced autoimmune diabetes in Chinese patient: a case report. Medicine (Baltimore). 2018 Nov;97(45):e12907.

8 Costa R, Carneiro BA, Agulnik M, Rademaker AW, Pai SG, Villaflor VM, et al. Toxicity profile of approved antiPD-1 monoclonal antibodies in solid tumors: a systematic review and meta-analysis of randomized clinical trials. Oncotarget. 2016 Nov 11;8(5):8910-20.

9 Herbst RS, Baas P, Kim DW, Felip E, Pérez-Gracia JL, Han JY, et al. Pembrolizumab versus docetaxel for previously treated, PD-L1-positive, advanced non-small-cell lung cancer (KEYNOTE-010): a randomised controlled trial. Lancet. 2016 Apr 9;387(10027):1540-50.

10 Vitt JR, Kreple C, Mahmood N, Dickerson E, Lopez GY, Richie MB. Autoimmune pancerebellitis associated with pembrolizumab therapy. Neurology. 2018 Jul 10;91(2):91-3.

11 Zurko J, Mehta A. Association of immune-mediated cerebellitis with immune checkpoint inhibitor therapy. Mayo Clin Proc Innov Qual Outcomes. 2018 Feb 1;2(1):74-7. 